\title{
3D-PRINTED WHOLE PROSTATE MODELS WITH TUMOR HOTSPOTS USING DUAL-EXTRUDER PRINTER
}

\author{
Kaisa Liimatainen $^{\star} \quad$ Leena Latonen $^{\dagger} \quad$ Kimmo Kartasalo $^{\star} \quad$ Pekka Ruusuvuori $^{\star}$ \\ $\star$ Faculty of Medicine and Health Technology, Tampere University, Tampere, Finland \\ $\dagger$ Institute of Biomedicine, University of Eastern Finland, Kuopio, Finland
}

\begin{abstract}
3D printing has emerged as a popular technology in various biomedical applications. Physical models of anatomical structures concretize the digital representations and can be used for teaching and analysis. In this study we combine 3D histology with 3D printing, creating realistic physical models of tissues with hotspots of interest. As an example we use mouse prostates containing tumors. Surface meshes are created from binary masks of HE-stained serial sections of mouse prostates and manually annotated tumor areas. Sections are interpolated to expand sparse image stacks for smoother results. Fiji, Meshlab and Tinkercad are used for mesh creation and processing. Objects are printed with Prusabased dual-extruder printer enabling different colors for tumors and the surrounding prostate tissue. Our 3D-printed mouse prostates appear realistic and tumors located at the edges of the organ are clearly visible. When transparent filament is used, the tumor hotspots are visible even when they are inside the prostate.
\end{abstract} eling

Index Terms - Models of organ physiology; Organ mod-

\section{INTRODUCTION}

3D printing is a technology for making physical models from three-dimensional representations of objects. Basically any model can be printed, with certain limitations for size and material when using printers for consumer use. In recent years, 3D printing technology has become a popular tool in biomedical research. Printing applications in medicine include, but are not limited to, printing tissues and organs with bioprinting systems, printing customized implants and prostheses with biocompatible materials, and printing anatomical models for surgical preparation $[1,2,3]$. 3D printing can also be used to visualize complex molecular structures for better understanding of underlying features [4]. In addition, printed models of anatomical and molecular structures are a novel resource for teaching of biomedicine and related fields $[5,6]$.

The most common imaging modality for acquiring anatomical data for 3D printing is computed tomography (CT), producing datasets in Digital Imaging and Communications in Medicine (DICOM) format [7]. DICOM data for models can also be acquired with magnetic resonance imaging (MRI), positron emission tomography (PET) and ultrasonography (US), to name a few [1]. However, to the best of our knowledge, 3D printing has not yet emerged as a tool for 3D histology with whole slide image (WSI) data. Sparse 3D histology data introduces new challenges for 3D modeling and printing when compared to e.g. CT or MRI data, where image stacks are more dense and natively aligned.

3D histology is a novel approach for digital pathology. In 3D histology, volumetric reconstructions are created from images of serial tissue sections [8]. Commonly, sections are hematoxylin-eosin (HE)-stained and imaged with light microscopy, producing a sequential stack of WSIs. 3D reconstruction techniques are then used to align image stacks for $3 \mathrm{D}$ representations [9].

In this study, we create models of mouse prostates from serial sectioned tissue. In addition, we model tumors in the tissue and combine this information to create a dual-color printed model. These physical models concretizing tumor locations and prostate anatomical shape can be used for e.g. teaching purposes and to provide complementary insight to quantitative analysis [10]. Even though anatomical models can be visualized and examined digitally, an actual physical model can still give an enhanced understanding of how the organ is formed and how the pathological lesions are situated. In addition, the model can be studied when powerful computers are not available, which can be the case in class room situations or when traveling.

\section{MATERIALS AND METHODS}

\subsection{Histology data}

Mouse prostates were fixed in PAXgene ${ }^{\mathrm{TM}}$ (PreAnalytiX $\mathrm{GmbH}$, Hombrechtikon, Switzerland) and formalin, followed by enclosing in paraffin and cutting into $5 \mu \mathrm{m}$ thick serial sections. The sections were placed on glass slides and HEstained. The slides were scanned with a pixel size of $0.46 \mu \mathrm{m}$ and stored in JPEG2000 format [11]. Tumors were annotated manually using the freehand tool in ImageJ [12]. One specimen consists of roughly 300 sections, with $1 / 10$ sections used 


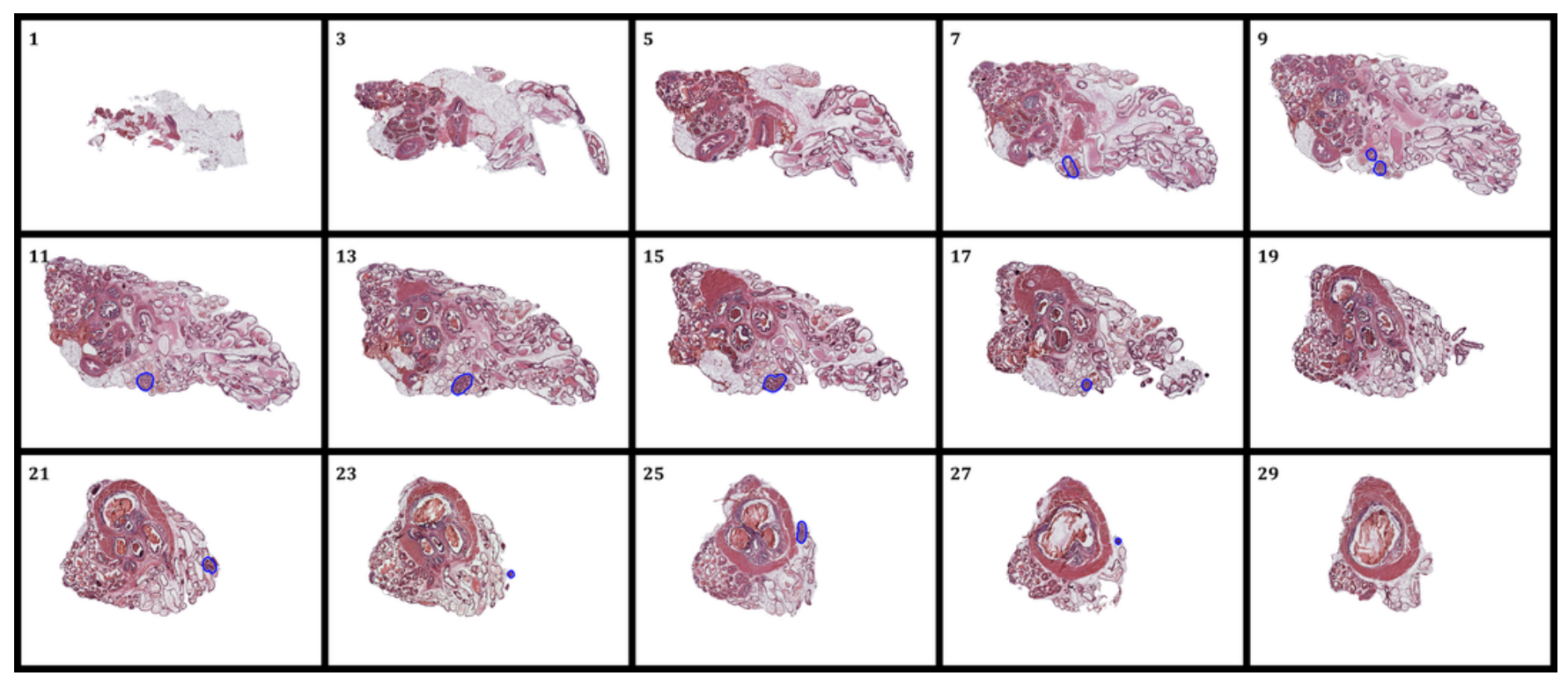

Fig. 1. Every second prostate section illustrated with tumor hotspots delineated in blue.

for HE staining and subsequent imaging. Thus, each of our image stacks has roughly $30 \mathrm{HE}$ stained sections scanned into WSIs, with a physical section-to-section distance of $50 \mu \mathrm{m}$. Use of mouse tissues in the research has been approved by the board of laboratory animal work of the State Provincial Offices of South Finland (licences ESAVI/6271/04.10.03/2011 and ESAVI/5147/04.10.07/2015).

\subsection{D reconstruction}

Initially, the sections have different relative orientations and locations in the WSIs, and a 3D reconstruction step is thus required to co-register all the images. This is accomplished by computing and applying a series of pairwise transformations estimated in a feature-based manner. We compute Scale Invariant Feature Transform (SIFT) [13] keypoints for each image pair and robustly fit affine transformations to the resulting point pairs [14]. We use an implementation of this algorithm provided in the TrakEM2 package [15] in Fiji [16]. The resulting transformations were also applied to binary masks indicating the tissue region and annotated tumors. In Figure 1, we show every second section from the reconstructed stack with tumor hotspots.

\subsection{Interpolation of virtual sections}

For constructing the 3D model, we use binary masks created from the registered sections. Since the image stacks are sparse with only 30 sections per stack, we interpolate virtual sections between the original sections using an iterative algorithm implemented in Python. To interpolate a new section between a pair of existing sections, we first perform an XOR operation between the two masks to obtain a new mask representing boundary regions only present on one of the original masks. We skeletonize this mask to get a new tissue boundary approximately equidistant from the two original boundaries. The region delineated by this boundary is then filled and any remaining branches are removed with binary erosion. The process is performed for all pairs of sections, and the resulting binary masks for new virtual sections are added to the stack. We performed the entire interpolation process three times, and applied it to both tissue and tumor masks, to obtain an equal number of sections for both models.

\subsection{Model creation and processing}

We create two separate triangular surface meshes, one for tissue (single object) and one for tumors (multiple objects). We import interpolated sections into Fiji as an image sequence, followed by conversion to binary format.

The height of the model is defined by changing the voxel depth parameter in the image properties. This value is calculated to approximately match the known aspect ratio of the data, taking into account the number of sections. At this point, the model size is also reduced so that it would fit better on the printing plate. We create a surface model using a resampling factor of 3 for tumors and 4 for tissue, and export it to a binary stereolithography (STL) model.

Meshlab is used to further process the STL files [17]. The number of faces is reduced with quadratic edge collapse decimation to make the model more manageable for 3D printing software. We apply various smoothing filters to get a smoother and, thus, a more realistic looking result.

Meshes are combined in Autodesk ${ }^{\circledR}$ Tinkercad $^{\mathrm{TM}}$ online service (https://www.tinkercad.com). First, a mouse identifi- 
a
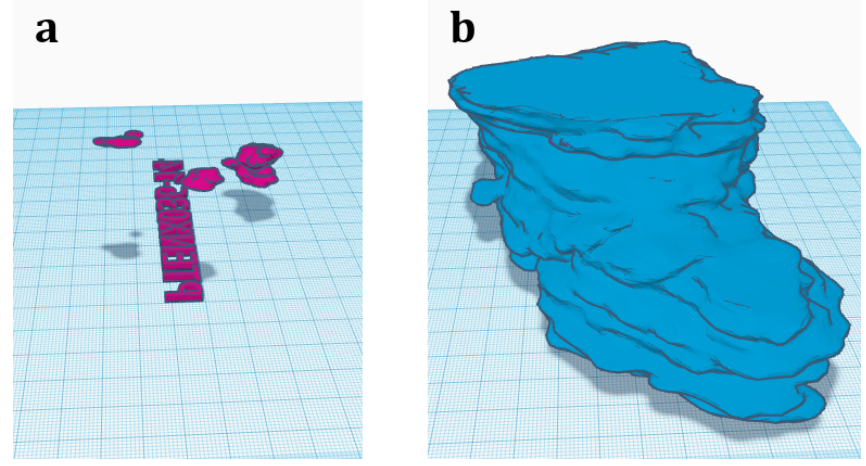

Fig. 2. Example illustration of finalized tumor (a) and tissue (b) models in Tinkercad.

cation number is added to the bottom of the tumor mesh, to be printed with the same color as the tumors. Then, to make the models fit together, the tumor mesh is subtracted from the prostate mesh to create holes at the locations of the tumors and the identification numbers. Example illustration of finalized tumor and tissue models in Tinkercad are shown in Figure 2. Finally, both meshes are repaired and imported to printing software which automatically combines the meshes.

\subsection{Printing the model}

We use a Prusa-based dual-extruder 3D printer and PLA and PETG filaments for printing. The model is transformed to g-code with Slic3r, and Repetier software is used to control the printer (https://www.repetier.com). Since we are dealing with a model of a biological origin, the shape is far from optimal for 3D printing. Thus, multiple parameters for infill and vertical shells (perimeter lines) were experimented with.

The models are printed with 25 to 100 percent infill and 2 to 7 vertical shells, depending on the size of the model and the filaments used. With solid colored filament we use low infill and just a few vertical cells. With transparent and translucent filaments 100 percent infill and high number of vertical shells are used for better transparency. The models are scaled between 20 to 60 percent of the original size to produce versions with different dimensions. However, the details of the tumors fade away in smaller sizes. It takes approximately one hour to print a small $4 \mathrm{~cm}$ long prostate, and three to four hours to print a large $7 \mathrm{~cm}$ long prostate. We use a $0.25 \mathrm{~mm}$ nozzle for tumors, and a $0.4 \mathrm{~mm}$ nozzle for the bulk of the tissue. A smaller nozzle is used for tumors to prevent leaking and to acquire a higher level of detail. Layer height was set to $0.2 \mathrm{~mm}$ for the first layer and $0.15 \mathrm{~mm}$ for other layers.

\section{RESULTS AND DISCUSSION}

Visualizations of 3D models in Meshlab, generated from the serial tissue sections with and without interpolation, are pre-

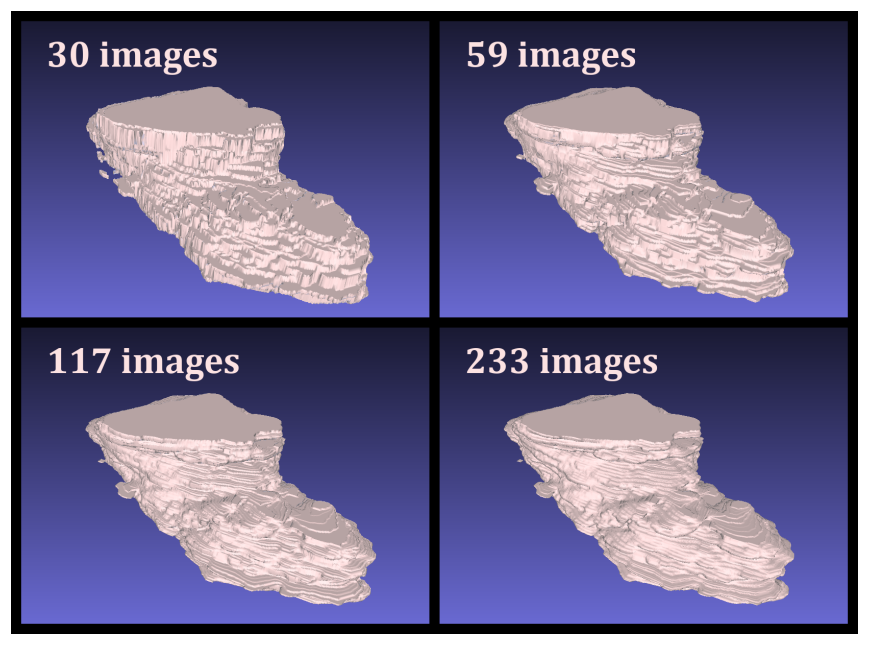

Fig. 3. Unprocessed prostate models in Meshlab after each iteration of interpolation. Only the original sections are used in the model at the top left.

sented in Figure 3. The models are triangular surface meshes that can be used in various applications. In this study, we used the models as designs for 3D printing, but the models could also be used as objects in 3D games or virtual reality. New sections were interpolated first between the original $30 \mathrm{sec}-$ tions and then iteratively between original and interpolated sections, resulting in approximately eight times as many sections as initially after the final iteration. With interpolation, we achieve a considerably more natural model when compared to using only the original 30 sections. We can see that without any interpolation the model would be very bulky, with blocklike shapes along the z-direction. With interpolation, the transition between sections becomes smoother. Furthermore, a larger number of interpolation iterations results in smoother models.

The sparsity of the data makes the models more prone to errors caused by e.g. distortions of individual physical sections. These errors might even be enhanced by interpolation, when the borders of one distorted section are far from surrounding sections. These distortions can be minimized with elastic transformation based $3 \mathrm{D}$ reconstruction methods that can fix the deformations. However, since tumor annotations were available for data reconstructed with SIFT keypoints, improving the reconstruction step was left out of the scope of this article. Quantitative error estimation of the SIFT keypoint based 3D reconstruction is presented by Kartasalo et al. [9]. Without native 3D imaging like $\mu \mathrm{CT}$, quantitative error estimation between 3D models and actual organ is non-trivial and out of the scope of this article.

In Figure 4 we show examples of 3D-printed mouse prostates with tumors, in different colors and sizes, representing two different specimens. We experimented with different filament types and colors. In the right column of Figure 4, 


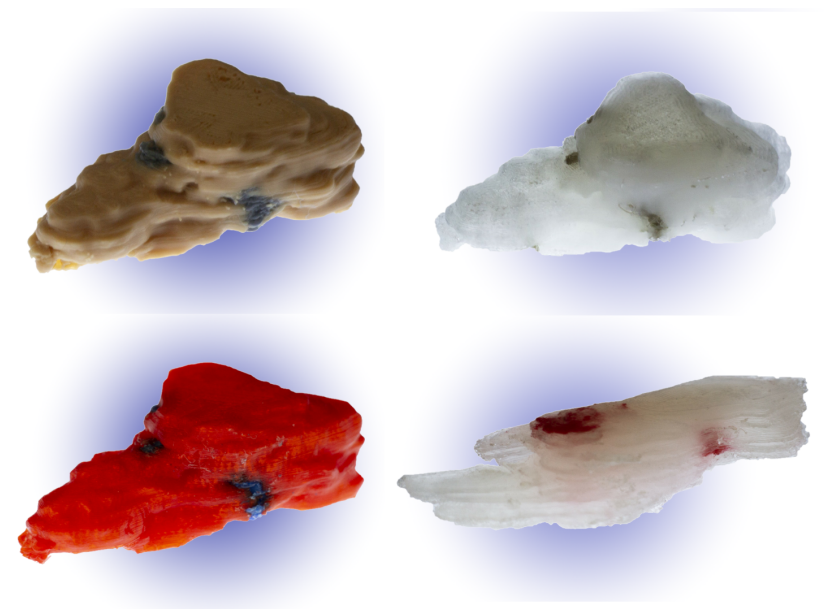

Fig. 4. 3D-printed models with solid color filament (left) and transparent (clear) filament (right). The tumor color shines through transparent models due to lighting conditions, slightly changing the otherwise clear color. The model on the bottom right represents a different specimen than the others.

prostate was printed with transparent filament and the tumors are clearly distinguishable. The visibility of the tumors is inferior in images on the left but prostate shape is more clear, especially with non-glossy filament (top left). Since the tumors are partly inside the tissue, there is a trade-off between good visibility of the tumors and the bulk tissue shape. When using transparent or translucent filaments for the tissue, the details of the tissue shape somewhat disappear due to missing shadows. On the other hand, if the tissue is printed with solid filament, the tumors inside the tissue cannot be seen. Fortunately, printing is low-cost and quite fast ( 0.5 to 4 hours per print, depending on printing parameters and size of the print), allowing several objects to be easily produced to highlight the morphological features of interest.

\section{CONCLUSIONS}

In this study, we created 3D-printed models of mouse prostates, including tumor hotspots. By applying section interpolation and mesh manipulations we succeeded in creating realistic and smooth prostate models based on only 30 tissue sections per prostate. We used a dual-extruder 3D printer, enabling the use of different filaments for prostatic tissue and tumors. With suitable colors and printing parameter optimization, we achieved clearly visible tumors within the prostate models.

\section{FUTURE WORK}

Further optimization is still required in order to produce the most informative and visually pleasant physical models of mouse prostates. Most tumors in the prostates used in this study lay on the outer edges of the prostate and, thus, are visible even without full transparency of the tissue model. However, tumors can be located inside the organ, and thus require transparency of the surrounding material to be visible. Achieving proper transparency for a 3D-printed model is possible, yet requires a lot of experimenting, since optimal parameters depend on the printer and filaments used.

In addition to tumors, we can model the inner structures of the tissue. In the case of prostate, these can be e.g. prostatic urethra and glands, highlighted with HE-staining. These structures can be manually annotated, but annotating multiple sections of many prostates would require considerable effort. Thus, automated methods for segmenting these structures will be studied. In addition, intelligent interpolation of virtual sections with generative adversial networks (GANs), for example, would provide more anatomically accurate models. After segmentation, a separate mesh for each structure of interest can be created similarly to the tumor meshes in this study. With a set of meshes representing tumors or any other pathological alterations, the normal anatomical structures, and the whole tissue, we can print very realistic organ models. We can also visualize specific properties computed from data. For example, we can model and print quantified features describing spatial heterogeneity [10]. All of the abovementioned models could also be imported to virtual reality environments to create an immersive experience of walking inside the tissue and viewing pathological lesions and computed features from a new perspective.

\section{ACKNOWLEDGEMENTS}

We thank Jukka Lehtiniemi and Iiro Leino for technical assistance.

\section{REFERENCES}

[1] F. Rengier et al., "3d printing based on imaging data: review of medical applications," Int. J. of Comput. Assisted Radiology and Surgery, vol. 5, no. 4, pp. 335-341, Jul 2010.

[2] C. Lee Ventola, "Medical applications for 3d printing: Current and projected uses," Pharmacy and Therapeutics, vol. 39, no. 10, pp. 704-711, 2014.

[3] P. Tack, J. Victor, P. Gemmel, and L. Annemans, "3dprinting techniques in a medical setting: a systematic literature review," BioMedical Engineering OnLine, vol. 15, no. 1, pp. 115, Oct 2016.

[4] MF. Coakley et al., "The nih 3d print exchange: A public resource for bioscientific and biomedical 3d prints," $3 D$ Printing and Additive Manufacturing, vol. 1, no. 3, 2014. 
[5] PG. McMenamin, MR. Quayle, CR. McHenry, and JW. Adams, "The production of anatomical teaching resources using threedimensional (3d) printing technology," Anatomical Sciences Edu., vol. 7, no. 6, pp. 479486, 2014.

[6] M. Kolitsky, "Reshaping teaching and learning with 3d printing technologies," e-mentor, vol. 4, no. 56, pp. 8494, 2014.

[7] D. Mitsouras et al., "Medical 3d printing for the radiologist," RadioGraphics, vol. 35, no. 7, 2015.

[8] D. Magee, Y. Song, S. Gilbert, N. Roberts, N. Wijayathunga, R. Wilcox, A. Bulpitt, and D. Treanor, "Histopathology in 3d: From three-dimensional reconstruction to multi-stain and multi-modal analysis," J. of Pathology Informatics, vol. 6, no. 6, 2015.

[9] K. Kartasalo, L. Latonen, J. Vihinen, T. Visakorpi, M. Nykter, and P. Ruusuvuori, "Comparative analysis of tissue reconstruction algorithms for 3d histology," Bioinformatics, vol. 34, no. 17, pp. 30133021, 2018.

[10] M. Valkonen, P. Ruusuvuori, K. Kartasalo, M. Nykter, T. Visakorpi, and L. Latonen, "Analysis of spatial heterogeneity in normal epithelium and preneoplastic alterations in mouse prostate tumor models," Scientific reports, vol. 7, pp. 44831, 2017.

[11] V. Tuominen and J. Isola, "Linking whole-slide microscope images with dicom by using jpeg2000 interactive protocol," J. Digit. Imaging., vol. 23, no. 4, pp. 454462, 2010.

[12] CA. Schneider, WS. Rasband, and KW. Eliceiri, "Nih image to imagej: 25 years of image analysis," Nature methods, vol. 9, no. 7, pp. 671, 2012.

[13] DG. Lowe, "Distinctive image features from scaleinvariant keypoints," Int. J. of Comput. Vision, vol. 60, no. 2, pp. 91-110, Nov 2004.

[14] MA. Fischler and RC. Bolles, "Random sample consensus: A paradigm for model fitting with applications to image analysis and automated cartography," Commun. $A C M$, vol. 24, no. 6, pp. 381-395, June 1981.

[15] A. Cardona et al., "Trakem2 software for neural circuit reconstruction," PLOS ONE, vol. 7, no. 6, 2012.

[16] J. Schindelin et al., "Fiji: an open-source platform for biological-image analysis," Nature methods, vol. 9, no. 7, pp. 676, 2012.

[17] P. Cignoni, M. Callieri, M. Corsini, M. Dellepiane, F. Ganovelli, and G. Ranzuglia, "MeshLab: an OpenSource Mesh Processing Tool," in Eurographics Italian Chapter Conf., Vittorio Scarano, Rosario De Chiara, and Ugo Erra, Eds. 2008, The Eurographics Assoc. 